\title{
DIETARY FIBRE AS PREBIOTICS IN NUTRITION
}

\author{
Emília Hijová, Izabela Bertková, Jana Štofilová \\ Institute of Experimental Medicine, Faculty of Medicine, P. J. Šafárik University in Košice, Košice, Slovak Republic
}

\begin{abstract}
SUMMARY
Trends in dietary nutrition and their personalization are progress in medical science and point out the necessity of adaptation and development of innovations in health system. The main objective of this article is to review the role of dietary fibre as prebiotics in nutrition with different functionality, its influence on modulation of intestinal microbiota, which has an essential role in maintenance of healthy organisms in people of all ages.
\end{abstract}

Key words: dietary fibre, prebiotic, nutrition, microbiome, health

Address for correspondence: E. Hijová, Institute of Experimental Medicine Faculty of Medicine, P. J. Šafárik University in Košice, SNP 1, 040 11 Košice, Slovak Republic. E-mail: emilia.hijova@upjs.sk

https://doi.org/10.21101/cejph.a5313

\section{INTRODUCTION}

Nutrition that constantly draws attention as one of major environmental factors influencing human health can be relatively easily influenced by an individual person as well as in the whole population. Recently, more emphasis has been placed on functional foods not only among professionals but also among lay people who are trying to live healthier under the influence of education training. Functional food can be defined as food that, in addition to classical ingredients, contains health benefits of ingredients which nutritional value has a beneficial effect on the health of the consumer, his physical and mental state. Effective components of functional food are in particular vitamins, minerals, probiotics, and prebiotics. The concept of probiotics and prebiotics is considered to be the most significant advancement in the field of nutrition and support of intestinal microbiota. It is the view that the composition of human food can be targeted and subsequently may selectively influence the composition of the intestinal microbiota and then the health of the organism.

\section{Prebiotics, Microbiome}

The term prebiotics was designed by professors Roberfroid and Gibson in 1995. Both were involved in the preparation of a functional food concept including prebiotics. Prebiotics are generally defined as "nondigestible food components that are resistant to the action of hydrolytic enzymes at the top of the GIT, pass into the colon in the unchanged state, and beneficially affect the microflora of the host organism by selectively stimulating the growth and/or activity of one or limited number of bacteria in the colon and thus improving the host health" (1).

This definition was updated in 2004 and prebiotics are now defined as "selectively fermented ingredients that allow specific changes, both in the composition and/or activity in the gastrointestinal microflora, that confer benefits upon the host well-being and health" (2).
The latest characterization of the human microbiome and its effect on health has led to a dramatic conceptual shift in research into the role of bioactive substances in the diet. Prebiotic foods prebiotics, which mainly include food fibre and resistant starches, are perceived as beneficial for maintaining healthy intestinal microbiota. However, the concept of human microbiome and its crucial role in human health and disease is newer, occurring in the 21 st century after arrival of the next generation of microbiome sequencing. Mapping diversity of the microbiome has unlocked many mysteries, but it also raises new questions. Answers on such many questions are still being missed, including a very basic but urgent question: "What diet is ideal for a healthy gut microbiome?" It remains unknown if there is an ideal intestinal microbiome that can be considered "healthy", or whether does it recognize an ideal diet that can positively modify the microbiome of people of different ages. In addition, there is a plethora of contradictory research results on some dietary components, therefore, the public can be confused whether they are healthy or not (3).

Research on human intestinal microbiota, referred to as the "forgotten organ" or "second brain", has exponentially increased in recent years with the latest advances in technology (4). There is an evidence that microbiota produces not only metabolites that can affect host physiology and these metabolites can also play an important role in the host's immune system and metabolism through a complex set of chemical interactions and signaling pathways (5-7). These interactions can greatly affect host health and disease risk (8) because the microbiota composition is associated with numerous diseases, e.g., irritable bowel syndrome (IBS), asthma, allergy, metabolic syndrome, diabetes, obesity, cardiovascular diseases, and colorectal cancer. In the last few decades there has been an increasing trend in the occurrence of metabolic diseases that affect the quality of life and represent a significant medical and economic burden on society. It is suggested that the diet is a major actor in the field of public health promotion as preventive measure to correct the risk of metabolic diseases. Decades of generalized nutritional recommendations has not led to mitigation of metabolic health crises. For long-term health maintenance 
there is currently no alternative healthy diet and recommendations for regular physical activity. Summarized evidence suggests that a more individual approach in disease prevention is needed and that such personalization cannot fully rely on human genomic variations in case of complex metabolic disease.

While taking into account these facts microbiome knowledge suggests that profiling the patient's microbiome will allow faster, more accurate and less invasive clinical decision-making process. In this context, prebiotics will be a component for individual adaptation of dietary interventions to change microbiome to a more effective configuration for disease prevention. Extensive characterization of the nutrition-microbiome-host metabolism axis will help to determine the integration of prebiotics and individual diet in the prevention of multifactorial metabolic diseases. Risk assessment of disease in susceptible populations remains one of the main goals of personalized and accurate nutrition, allowing identification of stratified subgroup in a way that improves the accuracy and cost-effectiveness of interventions and monitoring (examination). Additionally, early prognosis and/or diagnosis can facilitate prophylactic treatment that would otherwise be inappropriate for the majority of the population. For similar reasons, profiling a personalized (personal) microbiome is considered a promising tool for stratification of disease risk, not yet ready for use in clinical practice.

The system of predictive microbiome modelling integrates several aspects of intestinal flora such as the composition of the microbiota, quantity, metagenomics, metatranscriptomics, and metabolomics (9). In addition, biomarkers for assessing the microbiome for personalized prognosis, diagnosis and treatment may depend on geographic location, lifestyle, and many other factors. Therefore, if personalized profiling microbiome may be useful to be predictive for alleviation of disease, it will be a great scientific event and commitment before it will be ready for clinical settings.

\section{Dietary Fibre}

Dietary fibre has a long history, the term first applied by Hispley in 1953, and its definition has been revised, but the health benefits of high fibre foods have been long appreciated (10). In 430 BC, Hippocrates described the laxative effects of coarse wheat in comparison with refined wheat. In the 1920s, J. H. Kellogg published extensively on the attributes of bran, claiming it increased stool weight, promoted laxation and prevented disease (11). Dietary fibre was studied throughout the 1930s, and then forgotten until the 1970s. Denis Burkitt, the Irish physician and surgeon, is usually credited with re-popularizing the idea that dietary fibre protects against development of Western diseases, including diabetes, cardiovascular disease, colon cancer, and obesity (12). Since that time, research continues on defining fibre, measuring fibre, and determining the health benefits of fiber consumption (13).

EFSA defined dietary fibre as nondigestible carbohydrates plus lignin, including non-starch polysaccharides (NSP) - cellulose, hemicelluloses, pectins, hydrocolloids (i.e., gums, mucilages, $\beta$-glucans), resistant oligosaccharides - fructo-oligosaccharides (FOS), galactooligosaccharides (GOS), other resistant oligosaccharides, resistant starch - consisting of physically enclosed starch, some types of raw starch granules, retrograded amylose, chemically and/or physically modified starches, and lignin associated with the dietary fibre polysaccharides (14). The chemical classification of carbohydrates is usually based on molecular size and monomeric composition, three principal groups being sugars (1-2 monomers), oligosaccharides (3-9 monomers) and polysaccharides (10 or more monomers). Interest in the role of human intestinal microbiota in synergy with beneficial health effects associated with dietary fibre consumption is growing steadily. While the benefits of fibre, many of which have prebiotic properties, are well known, their mechanism of action usually remains a mystery. Without a knowledge of the relationship structure and function between different prebiotics and microbial species, as well as other considerations about microbial and host relationships, personalized and effective use of prebiotics for disease prevention will be difficult. Changing the composition of the intestinal microbiota can cause a number of factors, including changes in diet. Fibre consumption affects intestinal microbial flora by altering bacterial fermentation, colony size, and species composition (15).

Research activity in this area, containing data from human intervention studies on fibre and human intestinal microbiota, published in the literature from 1946 to 2016, were the basis for the creation of a new Systematic Review Data Repository (SRDR) $(16,17)$. From total of 188 distinct studies performed mostly in Europe the majority of them were randomised (96\%), and examined subjects were adults (more than 17 years of age) described as healthy (81\%). Studies have examined the effect of fibre on at least one of nine pre-defined areas of physiological health, namely:

- Total cholesterol and LDL cholesterol (lipid status);

- Post-prandial glucose and insulin (glycaemia and insulinemia); - Blood pressure;

- Faecal bulk and laxation;

- Transit time (the passage time of the stool by the intestinal tract);

- Bacterial fermentation and short chain fatty acids (SCFA) concentration;

- Modulation of colonic microbiota;

- Weight/adiposity;

- Increased satiety (appetite and saturation).

In Table 1 the fibre types used in studies are summarized, the most frequently studied were oligosaccharides ( $20 \%$ of studies), resistant starch (16\%), and chemically synthesized fibres (15\%), followed closely by inulin (13\%), bran (13\%), and cereal fibre $(11 \%)$. The results of the microbiology identified by the database have been examined in more detail and included in three categories: fermentation which includes short-chain fatty acid measurements, breath markers (such as hydrogen and methane), bacterial enzyme activity and metabolites, bile acid metabolism, and digestibility of fiber; bacterial composition comprising a relative or absolute number of bacteria; and $\mathrm{pH}$ of the colon and stool. The results of the database obtained from the interventional studies in terms of the dietary fibre-microbiota-physiology axis are shown in Figure 1.

Dietary habits shaped during the childhood and adolescence periods persist to adulthood and subsequently influence adult health. In addition to genetic predisposition to like sweet and salty flavours and dislike bitter and sour flavours and other innate, automatic mechanisms of appetite regulation, food preferences are shaped by the children's environment which is mostly created 
Table 1. Most commonly used prebiotics/fibre types in human studies (16)

\begin{tabular}{|c|c|}
\hline Group & Fibre types \\
\hline \multirow{5}{*}{ Oligosaccharide } & Fructooligosaccharide (FOS) \\
\hline & Galaktooligosaccharide (GOS) \\
\hline & Arabinoxylan-oligosaccharides \\
\hline & Xylo-oligosaccharide \\
\hline & Soybean oligosaccharides \\
\hline Resistant starch & Resistant starch type 1 , type 2 , type 3 , mixed \\
\hline \multirow{6}{*}{ Chemically synthesized } & Polydextrose \\
\hline & Dextrin \\
\hline & Soluble corn fibre \\
\hline & PolyGlykopleX (PGX) \\
\hline & Resistant starch type 4 \\
\hline & Microcrystalline cellulose \\
\hline Inulin & Inulin, oligofructose-enriched inulin \\
\hline Bran & Bran (wheat, oat, corn, barley, rye) \\
\hline Cereal fibre & Cereal fibre (wheat, barley, oat, rye) \\
\hline \multirow{7}{*}{$\begin{array}{l}\text { Fruit/vegetable/plant } \\
\text { fibres }\end{array}$} & Vegetable fibre \\
\hline & Lupin Kernel fibre \\
\hline & Sugar cane fibre \\
\hline & Sugar beet fibre \\
\hline & Bean fibre \\
\hline & Citrus fibre \\
\hline & Fruit fibre \\
\hline Combination & Combination/mixture \\
\hline Gums and mucilages & Gums, psyllium \\
\hline \multirow{5}{*}{$\begin{array}{l}\text { Non-starch } \\
\text { polysaccharides }\end{array}$} & Pectin \\
\hline & Cellulose \\
\hline & Hemicellulose \\
\hline & Beta-glucan, barley \\
\hline & Polysaccharide, non-starch \\
\hline High fibre diet & Dietary fibre \\
\hline
\end{tabular}

by their parents (18). The magnitude of changes taking place during adolescence, including the development of eating habits and changes in nutritional status, make this period critical for building good health and avoiding health consequences associated with improper nutrition. For example, the Polish observational study among adolescents $-1,565$ students aged $13-18$ years with normal weight, overweight and obesity - investigated the intake of dietary fibre and its sources according to prevalence of overweight after adjustment for age and gender (19). The intake of dietary fibre was not associated with the prevalence of overweight. The consumption frequency of dietary fibre and its sources was more related to the age and gender than to adolescents' weight. The role of dietary fibre in weight management is still uncertain. This study indicated that the general dietary pattern could be more important in the prevention of overweight than the separate intake of dietary fibre and its sources. Results are presented in adolescents, however, it is unknown how it is in adults. Additionally, obesity in childhood is strongly predictive of obesity in adulthood, therefore, for this reason again we return to the point that extensive characterization of the nutrition-microbiome-host metabolism axis may help to determine the integration of prebiotics and individual diet in the prevention of multifactorial metabolic diseases.

\section{Dietary Fibre and Health Benefits \\ Colonic Microbiota and Fermentation in Maintenance of Gut Health}

Prebiotics are non-digestible parts of food labelled carbohydrates that act as fibre. Unchanged they go to the colon where they are used by microorganisms of the intestinal microbiota, they become food for "good" intestinal bacteria and promote their growth, colonization and sustainability in the digestive tract. Among prebiotics are the most important oligosaccharides and galactooligosaccharides, which are referred to as bifidogenic substances (bifidofactors) with reference to their ability to selectively promote the growth of Bifidobacterium spp. (B. longum, B. breve, B. pseudolongum, B. infantis, B. lactis) and Lactobacillus spp. (L. acidophilus, L. casei, L. reuteri, L. plantarum).

Because there is a struggle about nutritional components in the intestine, the production of antimicrobial substances, ensur-

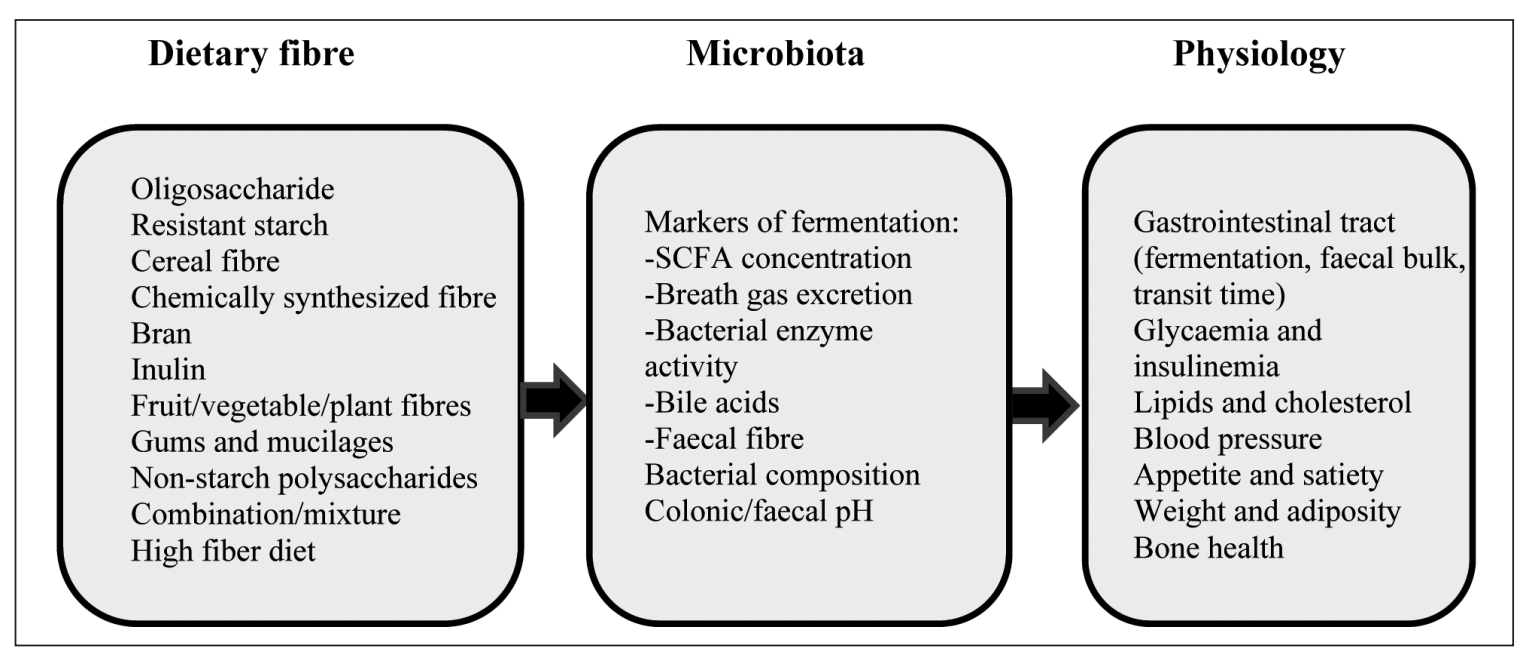

Fig. 1. Dietary fibre, microbiota, physiology axis (16). 
ing a sufficient amount of bifidobacteria and lactic acid bacteria, is essential for balanced intestinal microbiota. In the colon food components are fermented that were not fermented in the small intestine, e.g. hydrocarbons - carbohydrates (fibre, oligosaccharides, resistant starch, and the like). Protein residues, which can be further degraded by microbiota to toxic substances, nitrogen compounds (amines), phenolic compounds and branched chain acids are also passed through to the colon as a result of which it is necessary to stimulate the development and colonization of probiotic cultures in order to suppress the activity of proteolytic microbes. Without the presence of prebiotics in the diet, it would not be important to receive or use high doses of commercial probiotics. The fermentation of dietary fibre in the colon has a number of desirable attributes. The main product of polysaccharide fermentation in the colon is biomass, which not only increases stool bulk (20) but gives rise to increased numbers or metabolic activity of main saccharolytic bacterial species. Increased stool bulk reduces colonic transit time which is beneficial not only for the relief and prevention of constipation, but in reducing the impact of detrimental microflora associated characteristics such as toxic nitrogenous compounds, hydrogen sulphide, and production of carcinogenic or genotoxic compounds (21).

In the process of prebiotic fermentation in the large intestine SCFA is formed, such as acetic acid, propionic acid, butyrate, vitamin B12 and vitamin K, then absorbed by the intestinal mucosa and distributed by the vascular and lymphatic system to cells of organism (22). The resulting acetate is metabolized predominantly in muscle cells, kidneys, heart and brain. Propionate is used in the liver and is also a precursor for suppressing cholesterol synthesis. Butyrate is metabolized directly in the intestinal epithelium, where it serves as a regulator of cell growth and division. Short chain fatty acid production reduces the $\mathrm{pH}$ of the environment, thereby promoting growth and cell differentiation of intestinal epithelial cells and re-supporting the microflora. Fermentation in the intestine also produces the final form of the degradation of substances such as simple gases: hydrogen, carbon dioxide, hydrogen sulphide and methane.

Oligosaccharides are defined as glycosides with different degrees of polymerization, which can be synthesized chemically or enzymatically. The oligosaccharides include galactooligosaccharides (GOS), fructooligosaccharides (FOS), lactulose, raffinose derivatives - soy oligosaccharides, xylo- and chitooligosaccharides and inulin. Galactooligosaccharides produced from lactose by transgalactosylase $\beta$-galactosidase activity are called transgalactosylated oligosaccharides or transgalactooligosaccharides (trans GOS=TOS). Oligosaccharides naturally occur in breast milk (of which 90\% are GOS and 10\% FOS). Most commercially used are industrially produced prebiotics - galactooligosaccharides, which are most closely related to prebiotics commonly found in nature. Their health benefits can be described in two possible ways - the first step is to promote selective proliferation and attachment of probiotic bacteria to the intestinal epithelium (mainly Bifidobacterium spp. and Lactobacillus spp.), reducing space for pathogen colonization and thus decreasing exo- and endogenous intestinal infections. The second step is that the metabolic activity of probiotic bacteria triggers the cascade of the synthesis of beneficial substances and enzymes, it influences metabolic processes and prevents the onset of pathological processes in the organism. Prebiotic FOS and GOS naturally occur in cereal grains, onions, leeks, garlic, asparagus, bananas, raisins, chicory sprouts, soybeans, acacia gums, psyllium, and other plants (23).

\section{Prevention of Carcinogenesis}

The role of dietary fibre in preventing colorectal cancer (CRC) continues to be a topic of heated debate. Animal studies suggest that prebiotic inulin reduces the risk of $\operatorname{CRC}(24,25)$, but human studies have shown mixed results. For example, the 8-year Polyp Prevention Trial (PPT) evaluated the effects of high-fibre, high fruit and vegetable, and low-fat diet on the recurrence of adenomatous polyps in the colon (26). This study failed to show an effect of diet on adenoma recurrence after 8 years of follow-up. The lack of relationship between high-fibre diet interventions and colorectal cancer risk may be authentic, or it may be a product of the long latency period for colorectal cancer development.

Yao et al. (27) identified randomized controlled trials (RCTs) from specialized registers to assess the effect of dietary fibre on the recurrence of colorectal adenomatous polyps in people and on the incidence of colorectal cancer compared to placebo. The increased dietary fibre intake reduced the recurrence of adenomatous polyps in those with a history of adenomatous polyps within two to eight years. Long-term trials with higher dietary fibre levels are needed to enable confident conclusion because adenomatous polyp is a surrogate outcome for the unobserved true endpoint CRC.

\section{Impact on Cardiovascular System, Metabolic Syndrome, Dia- betes, Obesity}

Cardiovascular diseases remain the leading cause of mortality in the world. Epidemiological studies suggest that adequate fibre intake consistently lowers the risk of cardiovascular diseases and coronary heart disease $(28,29)$. Metabolic syndrome encompasses co-morbidities like obesity, dyslipidaemia, hypertension, insulin resistance, and hyperglycaemia, which increase the risk of cardiovascular diseases. A systematic literature review in four electronic databases and reference lists evaluate the effects of some prebiotics and synbiotics on inflammatory markers in adults with overweight or obesity. The authors concluded that some prebiotics and synbiotics may have immunomodulatory action, however, more randomized controlled trials are needed to support the clinical use of inulin-type fructans, galacto-oligosaccharides or related synbiotics for the treatment of metabolic endotoxaemia or low-grade inflammation in overweight/obese people (30). The effect of the fermentable fibre, resistant starch (RS4) on the gut microbial ecology in a free-living Caucasian cohort with signs of metabolic syndrome was investigated in the first holistic study. The findings showed that dietary RS4 induced changes in the gut microbiota are linked to its biological activity in individuals with signs of metabolic syndrome. These results have potential implications for dietary guidelines in metabolic health management (31).

Kondo et al. pointed out the cardioprotective effect of fibre-rich diet with brown rice improving endothelial function assessed by fasting flow debt repayment but without changes of total, HDL, and LDL-cholesterol, urine 8-isoprostane levels and $\mathrm{HbAlc}$ levels in patients with type 2 diabetes mellitus (32). In another clinical study the acute, post-prandial glycaemic and insulinemic response to a cookie containing resistant starch in the form of distarch phosphate (VERSAFIBE 1490 resistant starch) was as- 
sessed in healthy adults. This study showed that distarch can be incorporated into a cookie and significantly reduced post-prandial glucose and insulin responses in healthy adults (33).

\section{CONCLUSION}

Dietary fibre is an important food ingredient, and extensive characterization and gaining new insights into fibre activity on intestinal microflora for chronic diseases is important because of the possible profiling of a personalized microbiome, which is a promising tool for predicting and alleviating disease. Using a personalized microbiome in clinical practice from different perspectives will be a test of the future.

\section{Acknowledgement}

This project was supported by the Slovak Research and Development Agency under the project APVV-16-0176 and project with ITMS 26220120058 .

\section{Conflict of Interests}

None declared

\section{REFERENCES}

1. Gibson GR, Roberfroid MB. Dietary modulation of the human colonic microbiota. Introducing the concept of prebiotics. J Nutr. 1995;125(6):1401-12

2. Gibson GR, Probert HM, Loo VJ, Rastall RA, Roberfroid MB. Dietary modulation of the human colonic microbiota: updating the concept of prebiotics. Nutr Res Rev. 2004;17(2):259-75.

3. Dey M. Toward a personalized approach in prebiotic research. Nutrients. 2017;9(2):92-5

4. O’Hara AM, Shanahan F. The gut flora as a forgotten organ. EMBO Rep. 2006;7(7):688-93.

5. Clemente JC, Ursell LK, Parfrey LW, Knight R. The impact of the gut microbiota on human health: an integrative view. Cell. 2012;148(6):125870 .

6. Hooper LV, Littman DR, Macpherson AJ. Interactions between the microbiota and the immune system. Science. 2012;336(6086):1268-73.

7. Nicholson JK, Holmes E, Kinross J, Burcelin R, Gibson G, Jia $\mathrm{W}$, et al. Host-gut microbiota metabolic interactions. Science. 2012;336(6086):1262-7.

8. Flint HJ, Duncan SH, Scott KP, Louis P. Links between diet, gut microbiota composition and gut metabolism. Proc Nutr Soc. 2015;74(1):13-22.

9. Zmora N, Zeevi D, Korem T, Segal E, Elinav E. Taking it personally: personalized utilization of the human microbiome in health and disease. Cell Host Microbe. 2016;19(1):12-20.

10. Hispley EH. Dietary „fiber“ and pregnancy toxaemia. Br Med J. 1953;2(4833):420-42.

11. Slavin JL. Dietary fiber: classification, chemical analyses, and food sources. J Am Diet Assoc. 1987;87(9):1164-71.

12. Coffin CS, Shaffer EA. The host air and cold facts of dietary fibre. Can J Gastroenterol. 2006;20(4):225-56.

13. Slavin J. Fiber and prebiotics: mechanisms and health benefits. Nutrients. 2013;5(4):1417-35.

14. EFSA. Scientific opinion on dietary reference values for carbohydrates and dietary fibres. EFSA J. 2010;8(3):1462. doi: 10.2903/j. efsa.2010.1462
15. Flint HJ. The impact of nutrition on the human microbiome. Nutr. Rev. 2012;70 Suppl 1:S10-3.

16. Sawicki CM, Livingston KA, Obin M, Roberts SB, Chung M, McKeown NM. Dietary fiber and the human gut microbiota: application of evidence mapping methodology. Nutrients. 2017 Feb 10;9(2). doi: 10.3390/ nu9020125.

17. Livingston KA, Chung M, Sawicki CM, Lyle BJ, Wang DD, Roberts SB et al. Development of a publicly available, comprehensive database of fiber and health outcomes: rationale and methods. PLoS One. 2016 Jun 27;11(6):e0156961. doi: 10.1371/journal.pone.0156961.

18. Scaglioni S, Salvioni M, Galimberti C. Influence of parental attitudes in the development of children eating behaviour. Br J Nutr. 2008;99 Suppl $1: \mathrm{S} 22-5$.

19. Wuenstel JW, Wądołowska L, Słowińska MA, Niedźwiedzka E, Kowalkowska J, Kurp L. Intake of dietary fibre and its sources related to adolescents' age and gender but not to their weight. Cent Eur J Public Health. 2016;24(3):211-6.

20. Bosaeus I. Fibre effects on intestinal functions (diarrhoea, constipation and irritable bowel syndrome). Clin Nutr Suppl. 2004;1:33-8.

21. Gibson GR. Fibre and effects on probiotic (the prebiotic concept). Clin Nutr Suppl. 2004;1:25-31.

22. Hijová, E, Chmelárová, A. Short chain fatty acids and colonic health. Bratisl Lek Listy. 2007;108(8):354-8.

23. Vidová M, Hronská H, Tokošová S, Rosenberg M. Importance of prebiotic and probiotic: the role of galactooligosaccharides as prebiotic additives: a review. Potravinárstvo. 2013;7:28-35. (In Slovak.)

24. Hijová E, Bomba A, Bertková I, Strojný L, Szabadošová V, Šoltésová A. Prebiotics and bioactive natural substances induce changes of colonic microflora in cancerous rats. Acta Biochimica Polonica. 2012;59(2):271-4.

25. Hijová E, Szabadošová V, Štofilová J, Hrčková G. Chemopreventive and metabolic effects of inulin on colon cancer development. J Vet Sci. 2013;14(4):387-93.

26. Lanza E, Yu B, Murphy G, Albert PS, Caan B, Marshall JR, et al. The polyp prevention trial continued follow-up study. No effect of lowfat, high-fiber, high-fruit, and -vegetable diet on adenoma recurrence eight years after randomization. Cancer Epidemiol Biomarkers Prev. 2007;16(9):1745-52

27. Yao Y, Suo T, Andersson R, Cao Y, Wang C, Lu J, et al. Dietary fibre for the prevention of recurrent colorectal adenomas and carcinomas. Cochrane Database Syst Rev. 2017 Jan 8;1:CD003430. doi: 10.1002/14651858. CD003430.pub2.

28. Wolk A, Manson JE, Stampfer MJ, Colditz GA, Hu FB, Speizer FE, et al. Long-term intake of dietary fiber and decreases risk of coronary heart disease among women. JAMA. 1999;281(21):1998-2004.

29. Kim Y, Je Y. Dietary fibre intake and mortality from cardiovascular disease and all cancers: a meta-analysis of prospective cohort studies. Arch Cardiovassc Dis. 2016;109(1):39-54.

30. Fernandes R, do Rosario VA, Mocellin MC, Kuntz MGF, Trindade EBSM. Effects of inulin-type fructans, galacto-oligosaccharides and synbiotics on inflammatory markers in adult patients with overweight or obesity: a systematic review. Clin Nutr. 2017;36(5):1197-206.

31. Upadhyaya B, McCormack L, Fardin-Kia AR, Juenemann R, Nichenametla S, Clapper J, et al. Impact of dietary resistant starch type 4 on human gut microbiota and immunometabolic functions. Scientic Reports. 2016;6:28797. doi: 10.1038/srep28797.

32. Kondo K, Morino K, Nishio Y, Ishikado A, Arima H, Nakao K, et al. Fiberrich diet with brown rice improves endothelial function in type 2 diabetes mellitus: a randomised controlled trial. PLoS One. 2017;12(6):e0179869. doi: 10.1371/journal.pone.0179869.

33. Stewart ML, Zimmer JP. A high fiber cookie made with resistant starch type 4 reduces post-prandial glucose and insulin responses in healthy adults. Nutrients. 2017 Mar 5;9(3). doi: 10.3390/nu9030237.

Received February 7, 2018 Accepted in revised form July 9, 2019 